\title{
Individueller religiöser Pluralismus - Perspektiven für kulturpsychologische Forschung
}

\author{
Ulrike Popp-Baier
}

Eingegangen: 27. September 2021 / Angenommen: 12. Januar 2022 / Online publiziert: 15. Februar 2022 (C) Der/die Autor(en) 2022

Zusammenfassung Vor einigen Jahren plädierte der Soziologe Peter Berger für einen Paradigmenwechsel, um die Beziehung zwischen Moderne und Religion zu verstehen. Er schlug vor, die Säkularisierungstheorie durch eine Theorie des Pluralismus zu ersetzen und akzentuierte dabei zwei Formen des Pluralismus: Die Koexistenz verschiedener Religionen und die Koexistenz religiöser und säkularer Diskurse in der Gesellschaft wie auch im individuellen Bewusstsein. Linda Woodhead ergänzte diese zwei Formen des religiösen Pluralismus durch eine dritte Form der Dedifferenzierung, die sich durch das Aufweichen klarer Grenzen zwischen Religionen und zwischen dem Religiösen und dem Säkularen auszeichne. Sie sieht in allen drei Pluralismen Erklärungspotenzial, um den Anstieg der sogenannten nones in vielen postindustriellen Gesellschaften zu begreifen. In diesem Beitrag wird anhand von Beispielen aus empirischen Forschungsprojekten auf das analytische Potenzial der drei Pluralismen im Hinblick auf das Phänomen eines individuellen religiösen Pluralismus verwiesen und mit Vorschlägen für entsprechende kulturpsychologische Analysen verbunden.

Schlüsselwörter Theorie des Pluralismus · Drei Pluralismen · Religionen · Religiöse und säkulare Diskurse · Kulturpsychologie 


\title{
Individual religious pluralism-perspectives for cultural psychological research
}

\begin{abstract}
A few years ago, sociologist Peter Berger argued in favour of a paradigm shift in understanding the relationship between modernity and religion. He proposed replacing secularisation theory with a theory of pluralism, thereby accentuating two forms of pluralism: the co-existence of different religions and the co-existence of religious and secular discourses in society as well as in individual consciousness. Linda Woodhead complemented these two forms of religious pluralism with a third form of dedifferentiation, characterised by the softening of clear boundaries between religions and between the religious and the secular. She sees explanatory potential in all three pluralisms to understand the rise of the so-called nones in many postindustrial societies. In this article, examples from empirical research projects are used to point out the analytical potential of the three pluralisms with regard to the phenomenon of individual religious pluralism, and are combined with suggestions for corresponding cultural-psychological analyses.
\end{abstract}

Keywords Theory of pluralism $\cdot$ Three pluralisms $\cdot$ Religions $\cdot$ Religious and secular discourses · Cultural psychology

\section{Pluralisme religieux individuel - perspectives pour la recherche en psychologie culturelle}

Résumé Il y a quelques années, le sociologue Peter Berger a plaidé pour un changement de paradigme dans la compréhension de la relation entre la modernité et la religion. Il a proposé de remplacer la théorie de la sécularisation par une théorie du pluralisme, accentuant deux formes de pluralisme: la coexistence de différentes religions et la coexistence de discours religieux et séculiers dans la société ainsi que dans la conscience individuelle. Linda Woodhead a complété ces deux formes de pluralisme religieux par une troisième forme de dédifférenciation, caractérisée par l'adoucissement des frontières claires entre les religions et entre le religieux et le séculier. Elle voit dans ces trois pluralismes un potentiel explicatif pour comprendre la montée des soi-disant nones dans de nombreuses sociétés postindustrielles. Dans cet article, des exemples tirés de projets de recherche empiriques sont utilisés pour souligner le potentiel analytique des trois pluralismes en ce qui concerne le phénomène du pluralisme religieux individuel et sont combinés avec des suggestions pour des analyses correspondantes en psychologie culturelle.

Mots clés Théorie du pluralisme · Trois pluralismes · Religions · Discours religieux et séculiers · Psychologie culturelle

\section{Im Strudel von Pluralisierungen}

Als Jürgen Habermas (1985) seine Rede „Die Krise des Wohlfahrtsstaates und die Erschöpfung utopischer Energien“ unter dem Obertitel „Die neue Unübersichtlich- 
keit" veröffentlichte, wurde dieser Titel schnell zu einem geflügelten Wort. In diesem Aufsatz richtete sich Habermas auf Transformationsprozesse, die westliche Demokratien während des fundamentalen Wandels von der Industriegesellschaft zu einer postindustriellen Gesellschaft kennzeichnen und die zu tiefgreifenden kulturellen Veränderungen führen, wobei vor allem vielfältige Formen von Pluralisierung und Individualisierung eine Rolle spielen.

Für die empirische sozialwissenschaftliche Religionsforschung, die Religion vor allem als einen Teil dieser sich wandelnden Kultur in den Blick nahm, war „Unübersichtlichkeit“ aber zunächst kein Thema. Empirische Studien zum religiösen Wandel zirkulierten um die Grundfragen einer Abnahme von Religion (z.B. an gesellschaftlicher Relevanz), einer Zunahme von Religion (z. B. an Vitalität) oder einer Transformation der Religion (z.B. der sozialen Formen) bezogen auf analytische Perspektiven, die Säkularisierungsprozesse, Marktmodelle, Kapitalformen oder Spiritualisierungsprozesse konturierten und damit für die entsprechenden Koordinaten sorgten, an denen sich die Interpretation der Befunde der verschiedenen empirischen Studien und die entsprechenden Debatten orientieren konnten. Eine allgemein akzeptierte Antwort auf die genannten Grundfragen bezogen auf die zahlreichen quantitativen und die etwas weniger zahlreichen qualitativen Studien konnte bisher aber nicht gegeben werden (vgl. z. B. Bruce 2017; Heelas 2014; Pollack und Rosta 2017; Stolz 2020; Wilkins-Laflamme 2021; Yang 2012). ${ }^{1}$ Das lag nicht zuletzt auch an den unterschiedlichen Konzeptualisierungen von Religion und von Spiritualität, die in diese Studien und Debatten ihren Eingang fanden und um die noch stets trefflich gestritten wird (vgl. z.B., Bergunder 2020; Fedele und Knibbe 2020).

Nun gibt es m.E. im Hinblick auf die neueren Debatten um einen adäquaten Religionsbegriff und um eine akzeptable Konzeptualisierung von Spiritualität allerdings einen bemerkenswerten Unterschied: Während es bei den diversen Religionsbegriffen vor allem um analytische Konzepte geht, die u. a. von HistorikerInnen, ReligionswissenschaftlerInnen im engeren Sinne, SoziologInnen, AnthropologInnen und PsychologInnen vorgeschlagen und diskutiert werden, ist die jüngste Debatte um eine akzeptable Definition von Spiritualität vor allem auch darauf gerichtet, die Vielfalt an alltäglichen oder lebensweltlichen Verständnissen von Spiritualität zu rekonstruieren und angemessen auf den Begriff zu bringen (vgl. z. B. Hense et al. 2014). Diese Sensibilität in der empirischen Religionsforschung für eine neue Unübersichtlichkeit im Bereich des Religiösen zeigt sich auch noch in einer Vielzahl von Konzepten, die weniger als begriffliche Ordnungsleistungen und mehr als Anerkennung einer neuen Unordnung verstanden werden können, wobei dann Konzepte wie z. B. populäre Religion (Knoblauch z. B. 2009), vicarious religion (Davie 2007), fuzzy fidelity (Voas 2009), Verbuntung (Zulehner 2011), Multireligiosität (Berghuijs 2018), diffused religion (Cipriani 2017), nonreligion (Lee z.B. 2015) oder auch lived religion (z.B. Ammerman 2014) auf empirischen Studien beruhen oder empirische Studien angeregt haben, die lebensweltlicher Religiosität, Spiritualität oder Säkularität vor allem in ihrer Vielfalt und Veränderbarkeit Rechnung tragen wollen.

\footnotetext{
1 Die Literatur zu diesen Studien und Debatten ist inzwischen kaum mehr zu übersehen. Es wird daher hier nur auf einige Publikationen verwiesen, die einen Überblick zum Forschungsstand anstreben oder einflussreiche Debatten akzentuieren.
} 
Bemühungen, diese Pluralisierungen in das Säkularisierungsparadigma mit einzubeziehen (vgl. z. B. Bruce 2017; Voas 2009) setzte Peter Berger (2012, 2014, 2017) eine alternative Meta-Erzählung entgegen, nämlich ein Paradigma des Pluralismus², das von zwei Pluralismen auf zwei verschiedenen, aber miteinander zusammenhängenden Ebenen ausgeht: Zum einen gibt es den religiösen Pluralismus, d.h. verschiedene Religionen existieren nebeneinander - sowohl in der Gesellschaft als auch im individuellen Bewusstsein - und zum anderen gibt es den Pluralismus einer Koexistenz von religiösem Diskurs und säkularem Diskurs - ebenfalls sowohl in der Gesellschaft als auch im individuellen Bewusstsein. Nach Berger kennzeichnet vor allem der zweite Pluralismus die Moderne bzw. ist eine Folge von Modernisierungsprozessen. ${ }^{3}$

Linda Woodhead (2016, 2017a, b) sieht in Bergers Überlegungen eine vielversprechende Forschungsperspektive. Im Lichte ihrer eigenen empirischen Untersuchungen zu den sogenannten religiösen nones in Großbritannien, zu denjenigen, die in Umfragen angeben, ,,keiner Religion“ anzugehören, schlägt Woodhead allerdings eine Ergänzung zu Bergers zweifachem Pluralismus vor, die auch noch dem spätoder postmodernen Phänomen der Dedifferenzierung in den postindustriellen Gesellschaften Rechnung tragen soll. Die Grenzziehungen, die Bergers zwei Pluralismen noch implizieren, verlieren nach ihren Untersuchungen für viele nones an Relevanz und definieren für sie keine subjektiven Realitäten mehr (vgl. Woodhead 2016, S. 46). Entscheidend für diesen „dritten Pluralismus“, diese „,kulturelle Superdiversität“" (Woodhead 2017a), ist dann das Aufweichen der klaren Grenzen zwischen den verschiedenen, von Berger noch in Anspruch genommenen Kategorien der verschiedenen Religionen und des religiösen und säkularen Diskurses. In allen drei Pluralismen sieht Woodhead (2017a, S. 144) Erklärungspotenzial, um den Anstieg der nones, dieses Artefakts moderner Meinungsumfragen, in vielen postindustriellen Gesellschaften zu begreifen.

Viele SoziologInnen haben inzwischen das enorme Anregungspotenzial, das von Bergers Publikationen zu den vielen Altären der Moderne für die empirische Religionsforschung ausgeht, betont und auch für gesellschaftspolitische Analysen weiter konturiert (vgl. z. B. Berger 2017, Casanova 2018; Woodhead 2017a, b). Ich möchte in diesem Artikel nun vor allem auf das Anregungspotenzial der drei Pluralismen für psychologische Analysen eingehen, die dann - in der Terminologie von Berger - vor allem die Ebene des individuellen Bewusstseins betreffen. Dazu möchte

\footnotetext{
2 Berger (2014, S. 1) verwendet den Begriff „Pluralismus“ im Sinne von Pluralität oder Diversität, allerdings mit dem normativen Zusatz, dass von Pluralismus nur dann die Rede sein kann, wenn Menschen mit diversem ethnischen Hintergrund, verschiedenen Weltanschauungen und moralischen Überzeugungen friedlich zusammenleben und freundschaftlich miteinander verkehren. Ich folge hier Berger (und dann auch Woodhead) und verwende hier „Pluralismus“ ebenfalls im Sinne von Pluralität oder Diversität und nicht im Sinne einer Ideologie, obwohl m. E. der Begriff ohne Bergers normativem Zusatz für empirische Forschung besser geeignet wäre.

3 Peter Berger hat entscheidende Beiträge zur Säkularisierungstheorie geliefert und später genau diese Theorie auch scharf kritisiert. Seine neue Theorie oder sein neues Paradigma kann man vielleicht als eine Synthese seiner früheren Überlegungen und Studien bezeichnen, die man schlagwortartig folgendermaßen zusammenfassen kann: Modernisierung führt weltweit nicht zum Rückgang von Religion, sondern zu Pluralisierung. In diesem Kontext muss dann das neue Paradigma verankert und ausgearbeitet werden, wozu Berger seinem eigenen Verständnis nach nur erste Ansätze formuliert hat (vgl. Berger 2014).
} 
ich (kritisch) an eine konzeptionelle Klärung von Yang (2014) anschließen. Yang (2014) kritisiert, dass Berger nicht deutlich genug zwischen sozialem und individuellem Pluralismus unterscheide. Auch wenn Berger zu Recht die Interaktion zwischen Pluralismen auf gesellschaftlicher Ebene und Pluralismen auf der Ebene des individuellen Bewusstseins betone, müsse eine adäquate Differenzierung zwischen diesen beiden Ebenen einer adäquaten Konzeptualisierung einer derartigen Interaktion vorausgehen. Nach Yang (2014) bezeichne Pluralismus auf der individuellen Ebene eine persönliche Perspektive, Philosophie oder einen Lebensstil im Hinblick auf den Umgang mit multiplen Religionen im eigenen Denken und Fühlen, während auf dem sozialen Niveau Pluralismus eine soziale Konfiguration des Umgangs mit multiplen Religionen in einer bestimmten Gesellschaft bezeichne. Nach Yang (2014) könnte dann bei jemandem, der zwar multiple Religionen in einer Gesellschaft wahrnimmt und anerkennt, aber seinen eigenen Glauben als den einzig wahren sieht, Pluralismus auf der individuellen Ebene nicht konstatiert werden. Das sieht m. E. Peter Berger aber anders. Pluralismus, so Berger (2014, S. 32), multipliziert die Anzahl der Plausibilitätsstrukturen in der sozialen Umgebung eines Individuums. ${ }^{4}$ In diesem Zusammenhang bleibt dann ein Management des Zweifels, so Berger, keinem Gläubigen in einer modernen Gesellschaft erspart. Dabei kann dann z.B. eine fundamentalistische Orientierung als ein Projekt verstanden werden, das den Zweifel eliminieren will. Allgemein konstatiert Berger (2014, S. 32), dass Pluralismus auf der Ebene des individuellen Bewusstseins eher das „Wie“ des Glaubens als das „Was“ des Glaubens verändert hat. Was früher Schicksal war oder für selbstverständlich angenommen wurde, ist nun das Ergebnis einer überlegten Wahl. Zur Erläuterung seiner These verweist Berger (2014, S. 32) dabei auf das folgende Gedankenexperiment: Man stelle sich einen jungen konservativen Katholiken in den USA vor, dessen Familie katholisch ist, und der das sogenannte „Dritte - Generation - Phänomen“ zeigt: Er adoptiert die Glaubensüberzeugungen und Werte seiner aus Österreich eingewanderten Großeltern, welche die Eltern zurückgewiesen haben. Nun könnte man eine Tabelle mit zwei Spalten machen und in der einen Spalte die katholischen Überzeugungen und Praktiken der Großeltern auflisten und in einer Spalte diejenigen des Enkels. Die beiden Listen könnten nahezu identisch sein, und dennoch ist die Religiosität des Enkels radikal verschieden von der Religiosität der Großeltern, so verschieden, wie sich eben das Gewählte vom Selbstverständlichen unterscheidet. Dabei hat sich nicht das, was geglaubt wird, radikal verändert, sondern wie geglaubt wird. In diesem Zusammenhang bezieht sich Peter Berger (2014, S. 29) auch auf die von Alfred Schütz vorgenommene Unterscheidung verschiedener Bewusstseinsebenen im Hinblick auf Gewissheit, wonach die unterste Ebene diejenige der nicht in Frage gestellten Gewissheiten ist, des Selbstverständlichen und die oberste Ebene aus Präferenzen und Meinungen besteht, bei denen der Handelnde selbst annimmt, dass sie sich aufgrund entsprechender Erfahrungen oder auch guter Argumente ändern können. Nach Berger gehört es zu den Konsequenzen des Pluralismus, dass sich Religion auf den Bewusstseinsebenen des Individuums nach

\footnotetext{
${ }^{4}$ Casanova (2018) begründet übrigens seine besondere Wertschätzung dieser Publikation von Berger (2014) damit, dass Berger in diesem Buch wieder an seine früheren, an Alfred Schütz orientierten, phänomenologischen Analysen anschließt.
} 
oben bewegt hat: Vom Niveau des Selbstverständlichen zum Niveau der Optionen, Präferenzen und Meinungen.

Diese Überlegungen zeigen, dass der Terminus ,individueller religiöser Pluralismus“ doch noch anders verstanden werden muss, als Yang (2014) vorschlägt. Es handelt sich dabei letztlich um eine Kurzfassung des Ausdrucks ,individuelle Religiosität unter dem Einfluss von perzipiertem Pluralismus“" und nicht um die individuelle Adoption einer Ideologie, einer Philosophie oder eines Lebensstils, die dann nur einen Spezialfall eines derartigen individuellen religiösen Pluralismus darstellen würde. Im Folgenden werde ich Aspekte des individuellen religiösen Pluralismus akzentuieren, die bisher in (kultur-)psychologischen Analysen vielleicht noch nicht genügend beachtet wurden. Dazu werde ich Beispiele für das ,pluralistische Phänomen“ präsentieren. Bei diesen Illustrationen handelt es sich um Auszüge aus semistrukturierten oder narrativ-biografischen Interviews, die in den letzten Jahren im Zusammenhang mit verschiedenen Forschungsprojekten in den Niederlanden durchgeführt wurden. ${ }^{5}$ Sie werden bereits in der Form von formulierenden Interpretationen von Interviewsegmenten präsentiert, in denen zunächst - vor allem in indirekter Rede - die Wissensbestände, Deutungs- und Relevanzsysteme der InterviewpartnerInnen zusammenfassend rekonstruiert werden (vgl. Bohnsack 2000). Diese formulierenden Interpretationen werden allerdings auch schon mit ersten, den Orientierungsrahmen der InterviewpartnerInnen überschreitenden, reflektierenden Interpretationen verbunden (vgl. Kochinka 2008). Diese reflektierenden Interpretationen beziehen zum Teil auch die Ergebnisse anderer empirischer Studien oder theoretische Konzepte als Kontrasthorizonte mit ein. Dabei werde ich Linda Woodhead folgen und für die folgenden Ausführungen nur die Orientierungen von InterviewpartnerInnen berücksichtigen, die sich in den Interviews selbst keiner Religion zurechnen. ${ }^{6}$

\section{Individueller religiöser Pluralismus und kulturpsychologische Forschungsperspektiven}

\section{1 ,Einen Gott gibt es sicher ... die Frage ist nur welchen?“6}

Dass manche InterviewpartnerInnen, die angeben, keiner Religion anzugehören, damit manchmal auch meinen, sich nicht mit (nur) einer Religion verbunden zu fühlen

\footnotetext{
5 Es handelt sich dabei um narrativ-biografische Interviews im Zusammenhang mit einem eigenen Projekt zu religiösen und nichtreligiösen Orientierungen von jungen Erwachsenen mit einem höheren Bildungsabschluss und um semistrukturierte Interviews, die von Studierenden im Zusammenhang mit kleineren Projekten in Seminaren oder im Zusammenhang mit Abschlussarbeiten zu ähnlichen Themen durchgeführt wurden, wobei die InterviewpartnerInnen meistens auch Studierende waren. Die meisten Interviews wurden in niederländischer Sprache durchgeführt, einige wenige auch in Englisch. Die Namen der InterviewpartnerInnen sind selbstverständlich Pseudonyme. Alle Angaben, die zu einer Identifizierung der InterviewpartnerInnen beitragen könnten, wurden vage gehalten, manchmal verändert oder, wenn möglich, einfach weggelassen.

6 Nach den Angaben des CBS (Centraal Bureau voor Statistiek) rechneten sich 2019 ca. $54 \%$ der niederländischen Bevölkerung (die 15 Jahre oder älter ist) zu dieser Gruppierung. Im Jahr 2018 waren das noch ca. $52 \%$ und im Jahr 2012 nur ca. $46 \%$ (2021).
} 
oder zu beschäftigen und im Laufe der Interviews Elemente verschiedener religiöser Traditionen nennen, die für ihr Leben bedeutungsvoll sind, überrascht nicht. Es kommt immer wieder vor, dass für die eigene Lebensorientierung aus verschiedenen Quellen geschöpft wird und eine den individuellen Bedürfnissen entsprechende Partitur geschrieben wird. Diesem Phänomen der Multireligiosität wurden auch in den Niederlanden verschiedene Studien gewidmet. Joantine Berghuijs (2018) geht davon aus, dass etwa $23 \%$ der NiederländerInnen als multireligiös zu bezeichnen sind. Dabei ist allerdings nicht nur die Vielfalt der Kombinationen im Hinblick auf die inhaltlichen Orientierungen interessant, sondern auch die Vielfalt der entsprechenden Orientierungsleistungen, d. h. was vielleicht geglaubt oder nicht geglaubt, gehofft, erwartet, bevorzugt, befürchtet etc. wird.

Eine interessante Variante einer derartigen multireligiösen Orientierung kommt in einem semistrukturierten Interview mit einer Studentin zum Ausdruck, das im Rahmen einer Studie zum Gebrauch von mindfulness bei der Stressbewältigung durchgeführt wurde. Die Interviewpartnerin Bea gibt in diesem Interview an, dass die Bezeichnung ,religiös“ wohl für sie zutreffen würde, und sie bezieht diese Bezeichnung auf ihre Beschäftigung mit Religionen im alltagssprachlichen Sinn ${ }^{7}$, in ihrem Fall mit Spiritismus, dem Islam und dem Christentum. Diese ermöglichen ihr auch Copingstrategien, um sich zu beruhigen. Wenn sie nervös sei, bete sie ,auch schon mal“ zu Gott, zu Jesus oder zu Allah. Aber sie glaube „nicht definitiv“, dass auch einer von ihnen existieren würde. Etwas gebe es jedenfalls, es gebe jedenfalls einen Gott, aber sie wisse nicht genau, welchen. Bea bezeichnet sich selbst auch als ,jedenfalls spirituell“. Zur Kategorie „Spiritualität“ rechnet sie dabei verschiedene Praktiken, die paranormale Erfahrungen oder Einsichten ermöglichen (Handlesen, ein Medium besuchen, Tarot) oder mit östlichen Religionen in Zusammenhang gebracht werden (mindfulness, andere Meditationsformen). Mit diesen Praktiken hat sie aber erst vor kurzem begonnen. Diese verschiedenen Praktiken werden in verschiedenen Kontexten - auch im Alltag - häufig der Kategorie „Spiritualität“ zugeordnet (vgl. z. B. Bernts und Berghuijs 2016; Heelas und Woodhead 2005).

Auf die Frage nach ihrem Hintergrund im Hinblick auf Religion oder Spiritualität erzählt Bea, dass sie eigentlich erst vor ein paar Jahren religiös geworden sei, als sie sich mit einer Freundin mit Spiritismus beschäftigt habe und diese Beschäftigung dann über das Internet noch vertieft habe. Sie habe auch eine Freundin, die sei Muslimin, die habe ihr ,auch was erzählt“ und sie sei auch einmal mit in einer Moschee gewesen, was sie „schön gefunden“ habe. In die Kirche sei sie natürlich schon über die Schule gekommen - sie sei auf einer katholischen Grundschule gewesen - aber erst später habe sie auch die Kirche einen schönen Platz gefunden, als Gebäude, aber auch ,gefühlsmäßig“.

Hier wird deutlich, dass Bea Religiosität vor allem als eine kognitive Aktivität begreift, als eine explizite Beschäftigung mit Religionen, die dann aber auch mit

\footnotetext{
7 Bea ist m. E. wie vermutlich auch jede LeserIn dieses Artikels mit dem Religionsbegriff in dem Sinne vertraut, dass sie weiß, dass ,Religion“ vielfach als Allgemeinbegriff für buddhistische, jüdische, christliche, islamische, hinduistische, New Age und andere Traditionen verwendet wird, die dann auch als Religionen klassifiziert werden (vgl. Popp-Baier 2018). Dieses Religionsverständnis liegt auch den diversen Umfragen zugrunde, auf die in diesem Artikel bisweilen Bezug genommen wird und dieses alltagsweltliche Verständnis ist auch für die Argumentation in diesem Artikel ausreichend.
} 
emotional-ästhetischen Erlebnissen verbunden ist. Ihre Religiosität lässt Bea erst beginnen, als sie Spiritismus als „Lehre“ kennenlernt und sich damit intellektuell beschäftigt. Der Anreiz zu dieser Religiosität wird durch Sozialkontakte vermittelt. Dabei ermöglicht die neue Orientierung, die neue Religiosität, wohl auch eine ReInterpretation und eine Re-Evaluation älterer Orientierungs- und Erlebnisangebote: „Erst später“ wird auch die Kirche für sie zum ästhetischen und emotionalen Erlebnis. Dass für Bea Religiosität jedenfalls die explizite kognitive Beschäftigung mit Religion bedeutet, wird auch später im Interview noch deutlich, als sie davon spricht, dass sie immer schon an Geister geglaubt habe, auch schon bevor sie religiös wurde.

Anders verortet Bea Spiritualität in ihrer Lebensgeschichte. Spirituell, sagt sie, sei sie immer schon gewesen. Ihre Mutter sei auch spirituell, ihr Bruder habe als Kind „das zweite Gesicht“ gehabt. Sie wisse es ,eigentlich nicht so gut“, das Spirituelle sei ,eigentlich“ immer schon da gewesen. Damit ist Spiritualität vor allem das, was „immer schon da war“ und zum vertrauten Familienhintergrund gehört, auch wenn dann noch später erworbene Praktiken dieser Kategorie zugerechnet werden. Und dieses Vertraute besteht vor allem aus Paranormalem.

Im Laufe des Interviews erwähnt Bea noch, dass sie sich später vielleicht auf eine Religion beschränken möchte, aber sie wisse noch nicht auf welche. Das scheine ihr dann einfacher zu sein. Dann könne sie sich an eine Lebensweise halten. Andererseits finde sie es nun ,gut“, dass sie selbst auswähle. Sie wisse es eigentlich nicht.

Die spätere Entscheidung für eine der vielen möglichen Religionen gehört also zu ihrem Orientierungshorizont. Dem möglichen Vorteil der Komplexitätsreduzierung in der Zukunft wird dabei die Wahlfreiheit in der Gegenwart gegenübergestellt. Die Alternative, diese religiösen Orientierungen später vielleicht einmal aufzugeben, der Weg einer individuellen secular transition (vgl. z. B. Voas 2009) gehört jedoch nicht zu dem von ihr artikulierten Möglichkeitshorizont, denn ,einen Gott gibt es sicher“.

Die Typik, die man an diesem Beispiel einmal tentativ herausarbeiten kann, ist eine Verschränkung zwischen einem pragmatischen Pluralismus, einer ,Theodiversität“ (vgl. Norenzayan 2016) und einem ontologischen Monismus, einem Monotheismus im Hinblick auf die Gottesfrage vor dem selbstverständlichen Hintergrund paranormaler Praktiken und Erfahrungen. Man kann hier auch von einer Verschränkung von Sicherem (es gibt einen Gott), mit Unsicherem (welcher von den vielen Göttern ist der ,wahre“ Gott) mit Selbstverständlichem (paranormale Praktiken und Erfahrungen) sprechen. Derartigen Verschränkungen in ihrer kognitiv-pragmatischemotionalen Komplexität und ihren lebensgeschichtlichen und soziokulturellen Bezügen nachzuspüren, sollte noch stärker als Aufgabe kulturpsychologischer Analysen angesehen werden als es bisher vielleicht der Fall war.

\section{2 ,Yoga hat mich im Kopf beruhigt und es war eine angenehme Entspannung für meinen Körper 6}

Der von Berger konstatierte ,zweite Pluralismus“, die Koexistenz von religiösen und säkularen Diskursen im individuellen Bewusstsein, lässt sich unter anderem auch im medizinisch-therapeutischen Bereich zeigen. Praktiken wie Yoga oder mindfulness gehören inzwischen zum populären Repertoire von Entspannungstechniken, angebo- 
ten nicht nur auf einem gut florierenden Markt für gesundheitsbezogene Freizeitaktivitäten, sondern auch in so verschiedenen institutionellen Kontexten wie Krankenhäusern, Schulen, Universitäten oder Betrieben. Dabei werden diese Aktivitäten von manchen Praktizierenden als rein körperliche Übungen oder therapeutische Praktiken verstanden, manche verstehen sie als religiöse Aktivitäten, andere wiederum als spirituelle, manche als hybride Formen, manche verändern ihr Verständnis, während sie Yoga oder mindfulness praktizieren und manchen sind derartige mögliche Unterscheidungen oder Klassifikationen auch einfach gleichgültig.

Das oben angeführte Zitat stammt von einer Interviewpartnerin, die an einer Studie zu Motiven, um Yoga zu praktizieren, teilgenommen hat (vgl. Foe-Aman 2021). In dem Interview erzählt Anneke, dass sie nach ihrem Umzug in eine andere Stadt mit einer Yogadozentin Bekanntschaft geschlossen und dann auch bei ihr Unterricht genommen habe. Anfangs habe sie nicht viel über Yoga gewusst, es habe sie „,mental beruhigt“" und „körperlich entspannt“. In den folgenden Jahren habe sie sich dann aber mehr und mehr in Yoga vertieft. Sie wisse, dass Yoga aus Indien komme, so viel wisse sie vom Hinduismus, und sie mache nun vor allem ayurvedische Yoga. Sie interessiere sich sehr für Ayurveda, den Ursprung, die Heilkunde, und da gehöre auch Yoga dazu. Später betont Anneke noch einmal, dass Yoga für sie wichtig sei, weil Yoga für „Balance“ in ihrem Leben sorge, sie durch Yoga mental „,zur Ruhe“ komme und sie ihren Körper ,geschmeidig“ halten könne. Die Frage des Interviewers, ob Yoga zu ihrer persönlichen Entwicklung, ihrer Selbstkenntnis, Selbstaktualisierung oder Autonomie beitragen würde, verneint Anneke. Jedoch sehe sie Yoga als Teil ihres spirituellen Weges. Sie finde, dass, ,gut für den eigenen Körper zu sorgen“, viel zu wenig Aufmerksamkeit geschenkt werde. Sie glaube daran, dass das Praktizieren von Yoga den „Kontakt mit ihrer Weiblichkeit“ vergrößere und dass die „weibliche Kraft“" sehr wichtig sei. Sie glaube, dass Ayurveda und Yoga nicht ohne Grund ,ihren Weg gekreuzt“ hätten, dass sie ihr in ihrem Leben helfen würden, und das hoffe sie auch wieder an andere weiterzugeben. Es sei Teil ihres spirituellen Wachstums, sie ,glaube an“ Energien, den Stand des Mondes, die Jahreszeiten und die vier Elemente. Diese müssten wir in unser tägliches Leben integrieren und das würde uns im Leben helfen. ${ }^{8}$

Auch wenn Anneke Yoga zunächst über Sozialkontakte kennenlernte und dabei zunächst vor allem den therapeutischen Wert dieser Praktik schätzte, hat sie schließlich Yoga in ihre Weltanschauung bzw. ihre „Spiritualität“ integriert oder auch ihre „Spiritualität“ im Zusammenhang mit einer derartigen Praktik entwickelt, auch wenn sie noch stets die therapeutischen und schließlich auch pädagogischen Folgen dieser Praktik betont.

Ganz anders ein Yogalehrer, der sich aufgrund seiner bisherigen Erfahrungen mit seinen SchülerInnen eine bessere Nutzung seines Angebots dadurch ausrechnete, dass er alle möglichen spirituellen Assoziationen mit dieser Praktik ausschaltet

\footnotetext{
${ }^{8}$ Diese formulierende Interpretation bezieht sich auf das Interviewtranskript 1 im Anhang zu Foe-Amans (2021) Abschlussarbeit für den Bachelor Religionswissenschaften.
} 
und der sich deshalb entschloss, seine Yogaklassen umzubenennen in Stretchen met Ferdy. ${ }^{9}$

Während man bei Anneke nun im Lichte von Bergers zweitem Pluralismus eine Koexistenz der säkularen Diskurse der Pädagogik und der Therapie mit einem Diskurs des Spirituellen konstatieren kann, könnte man bei dem genannten Yogalehrer wohl von der Koexistenz eines Sport-Diskurses sprechen, der den „religiösen“ Diskurs in manchen Kontexten (strategisch) überlagert bzw. ersetzt. Ebenso hilfreich für eine Akzentuierung derartiger Orientierungen wäre wohl Woodheads dritter Pluralismus, ihre Konstatierung einer Dedifferenzierung, denn die Grenzen dieser Diskurse sind in der Tat vage. Auch in diesen und anderen Fällen könnten kulturpsychologische Analysen dieser ,,kulturellen Superdiversität“ nachspüren und plurale Orientierungsmuster aus der Perspektive der Handelnden in ihren lebensgeschichtlichen und soziokulturellen Kontexten in den Blick bringen. ${ }^{10}$

\section{3 ,... ich versuche als ein guter Mensch zu leben ... und das bekomme ich dann auf die eine oder andere Weise auch zurück ...."}

Diese Einschätzung artikuliert ein junger Student in einem narrativ-biographischen Interview zu der allgemeinen Leitfrage, ob und inwiefern Religion oder Spiritualität eine Rolle in seinem Leben spielen würden. Allert bezeichnet sich selbst als spirituell. Er ist reformiert aufgewachsen, aber nicht mehr mit der reformierten Kirche und auch mit keiner anderen Religionsgemeinschaft verbunden. Er hat ,ein bisschen mit Drogen experimentiert“ und hatte ,einige gute Erfahrungen mit XTC“, die er als spirituelle Erfahrungen begreift. Auch mit Musik verbindet Allert spirituelle Erfahrungen. Allert lebt in einer Wohngemeinschaft. Der junge Mann, der vor ihm in seinem Zimmer wohnte, hatte sich das Leben genommen. Das war ein ziemlicher Schock für Allert. Allerts oben angeführte „Lebensreflexion“ steht in einem Artikulationszusammenhang, den ich wegen seiner semantischen Komplexität doch einmal wörtlich zitieren möchte ${ }^{11}$ :

Ja - ich will nicht sehr arrogant tun - ich denke - als ich da nun darüber nachdenke - dann denke ich, nun ja, ich habe es - besser getroffen als viele andere Menschen auf der Welt - und dann denke ich, ja, vielleicht hat jemand doch - vielleicht hat jemand doch so etwas wie, nun der Junge ist so - das ist so ein netter Junge, oder so was ((lacht)) und der Junge meint es auch so gut,

\footnotetext{
9 Den Hinweis auf den entsprechenden Artikel im Rotterdams Dagblad im Jahr 2016 verdanke ich Lotje Vermeulen, die diesen Artikel auch bereits in einer Seminararbeit zitierte. Auf der website terracacti.nl/2017/10/yoga-zonder-poespas (zuletzt besucht am 23. September 2021) verweist besagter Yogalehrer noch einmal auf diese Namensänderung seiner Yogaklassen im Jahr 2016.

10 Im Falle von Praktiken wie Yoga wäre auch insbesondere eine Verschränkung von religionspsychologischen mit sportpsychologischen Perspektiven empfehlenswert. Zu einer diesbezüglichen kulturhistorischen Argumentation vgl. z. B. Alter (2006), der auf die Entwicklung von Yoga als ein system of metaphyical fitness am Ende des 19. und Beginn des 20. Jahrhunderts aufmerksam macht.

11 Die deutsche Übersetzung des Zitats ist von mir. Dabei wurden folgende Transkriptionsregeln verwendet ,," bezeichnet eine kurze, gerade noch wahrnehmbare Pause, ,-“"bezeichnet eine deutliche Pause und zwischen $((. .)$.$) werden parasprachliche Äußerungen vermerkt.$
} 
der verdient daher auch was - ja, ich weiß es nicht, aber - was ich auch gerade gesagt habe, ich versuche als ein guter Mensch zu leben - ich habe das Gefühl, dass ich das wohl sagen darf, und das bekomme ich dann auf die eine oder andere Weise auch zurück - und ja - ob da nun eine Zwischenperson etwas damit zu tun hat oder ob das eine direkte Wechselwirkung ist - das weif ich nicht - das weiß natürlich niemand, aber ich denk es - nein, ich denk es, ich denk es nicht, und wenn es jemand ist dann ist es denke ich zum Beispiel ein Opa, mein Opa oder so, oder jemand der wichtig für mich war, der gestorben ist - dass zum Beispiel mein Mitbewohner so etwas hat wie, nun, äh, ich gönne das dem Jungen - so etwas, und solche Dinge kann ich schon denken - für den Rest denke ich nicht, äh-nicht eine Person die ich dann nicht kenne, das geht mir dann wieder etwas zu, zu-wird mir schon wieder etwas zu abstrakt glaube ich

Dieses Interviewsegment kann man als eine Artikulation (von Aspekten) eines sogenannten „Gerechte-Welt-Glaubens“ verstehen, nach dem „Menschen bekommen, was sie verdienen und verdienen, was sie bekommen“ (vgl. dazu Lerner 1980). ${ }^{12}$ Allert artikuliert diesen Glauben als eine auf seine eigene Person bezogene Intuition, zu deren Plausibilisierung er quasi seine religiöse Phantasie bemüht und mit diversen Erklärungsoptionen jongliert. Allert präsentiert sich in diesem Interviewsegment als jemanden, der erfahren hat, dass es ihm besser geht als anderen Menschen und diesen Umstand bezieht er intuitiv auf seine ,guten“ Intentionen und Handlungen. Für eine Erklärung dieser Beziehung werden dann verschiedene Optionen in Betracht gezogen, manche werden dabei auch verworfen. Die Grundfrage, ob es da eine unmittelbare Beziehung gebe oder ob, wie er sagt, eine Zwischenperson, damit etwas zu tun habe, bleibt dabei offen. Allert imaginiert in diesem Interviewsegment einen möglichen Einfluss eines Verstorbenen, seines Opas oder seines verstorbenen Mitbewohners. Dabei geht es auch weniger um religiösen „Glauben“ als um diverse religiöse Imaginationen, um mögliche religiöse Interpretationen, die für Allert eine gewisse Stimmigkeit im Hinblick auf seine Intuitionen, sein Lebensgefühl und mögliche biografische Kohärenzen aufweisen.

Kognitionswissenschaftliche Studien haben in den letzten Jahrzehnten deutlich gemacht, wie moralische Intuitionen und implizite Annahmen zur Logik sozialer Interaktionen religiöses Denken und Orientieren für das Individuum plausibilisieren können (vgl. z. B. Boyer 2001). Andere Studien legen nahe, dass unsere Orientierungen, Schlussfolgerungen und Entscheidungen im Alltag eher von impliziten Heuristiken und Gewohnheiten bestimmt werden als von rationalen Überlegungen und Schlussfolgerungen, die den Gesetzen der Logik folgen (vgl. z. B. Kahnemann

\footnotetext{
12 Das Interview mit Allert gehört zu einer Studie zu religiösen oder nichtreligiösen Orientierungen von jungen Erwachsenen mit höherem Bildungsabschluss in den Niederlanden. In den dabei durchgeführten 34 narrativ-biografischen Interviews artikulierten 18 InterviewpartnerInnen einen Gerechte-Welt-Glauben, drei InterviewpartnerInnen vergleichbare Perspektiven und in fünf Interviews wurden die Lebensreflexionen der InterviewpartnerInnen von moralischen Überzeugungen dominiert, die vor allem auch gerechtigkeitsbezogene Argumentationen umfassen. Diese Angaben beziehen sich auf den jetzigen Stand der Auswertung der Daten, die noch nicht abgeschlossen ist.
} 
2012). ${ }^{13}$ Rationales Denken und Handeln bedarf der motivierten Entscheidung, sich den entsprechenden Anstrengungen des Denkens zu unterziehen und eventuell auch die rationalen Formen dieses Denkens im Alltag einzuüben und zu verankern. Anthropologische Studien haben gezeigt, dass die Akzeptanz von wissenschaftlichen Erklärungen von bestimmten Ereignissen (z.B. Erklärungen von Krankheiten als von Bakterien oder von Viren verursacht), ,religiöse“ Erklärungen (z. B. Erklärungen von Krankheiten aufgrund von dämonischen Einflüssen oder aufgrund von Hexerei) nicht einfach ersetzen, sondern dass häufig Kombinationen bevorzugt werden, bei denen die ,wissenschaftlichen“ Erklärungen die anderen Erklärungen ergänzen, überlagern oder auch kontaminieren können (vgl. z. B. Legare und Gelman 2008; Lanman 2016; aber auch schon Evans-Pritchard 1937). Derartige Kombinationen können durchaus in eine gelungene Alltagspraxis integriert werden. ${ }^{14}$ Bergers und Woodheads Pluralismen können für das Aufspüren derartiger Koexistenzen sensibilisieren und entsprechende kulturpsychologische Studien könnten dann vor allem auch versuchen, das komplexe Zusammenspiel von Intuitionen und Reflexionen zu analysieren - unter Berücksichtigung eines ,kognitiven Unbewussten“ wie auch eines in einem transgenerationalen und soziohistorischen Kontext verankerten ,dynamischen Unbewussten“ (vgl. zu letzterem Straub und Tepeli 2021).

\section{Fazit}

In ihrem Buch God in Nederland 1966-2015 werten die AutorInnen Ton Bernts und Joantine Berghuijs (2016) eine Umfrage zur christlichen Religion in den Niederlanden aus, die seit 1966 fünfmal durchgeführt wurde. Dabei wurden in dieser Publikation von 2016 die Ergebnisse der Umfrage von 2015 präsentiert und ihre Relationen zu den früheren Umfragen diskutiert. Die AutorInnen fassen ihre Ergebnisse folgendermaßen zusammen:

„Es wird deutlich, dass Glaube, Spiritualität und Sinngebung in verschiedener Hinsicht ,grenzenlos` geworden sind. Kirchenmauern markieren nicht mehr die Grenzen zwischen Gläubigen und Nichtgläubigen. Grenzen zwischen religiösen Traditionen wie auch Grenzen zwischen dem Religiösen, dem Spirituellen und dem Sä-

\footnotetext{
13 Gendler (2008) hat in diesem Zusammenhang das Konzept alief eingeführt, um für assoziative, automatische und arational Kognitionen zu sensibilisieren, die unser Verhalten und unsere Präferenzen im Alltag mitbestimmen.

${ }^{14}$ Legare und Gelman (2008) zitieren eine Geschichte des legendären Arztes Paul Farmer, der sich vor allem auch für die Entwicklung eines modernen Gesundheitssystems bei den ärmsten Nationen einsetzte. Diese Anekdote möchte ich den LeserInnen dieses Artikels nicht vorenthalten. Es geht um ein Gespräch Farmers mit einer älteren Haitianerin, die an Tuberkulose litt. In einem früheren Interview zeigte sich diese Frau beleidigt, als man sie nach einem etwaigen Glauben an Zauberei fragte. Sie antwortete, dass sie nicht dumm sei, dass sie wisse, dass Tuberkulose daher komme, dass Menschen sich mit Keimen infizierten. Sie hatte auch alle ihre Medikamente eingenommen und war geheilt worden. Als sie ein Jahr später noch einmal interviewt wurde, sagte sie, dass sie selbstverständlich an Zauberei glaube. Sie wisse auch, wer ihr damals diese Krankheit geschickt habe und sie werde es dieser Person noch heimzahlen. Auf die völlig entgeisterte Reaktion des Arztes, warum sie denn dann überhaupt ihre Medikamente eingenommen habe, antwortete sie etwa das Folgende: „Honey, are you incapable of complexity?“ (Kidder 2003 zitiert nach Legare und Kelman 2008, S. 607).
} 
kularen lassen sich nicht mehr deutlich ziehen“ (Bernts und Berghuijs 2016, S. 14). ${ }^{15}$ Weiterhin ergebe sich ein komplexes, aber auch schwer fassbares Feld von Spiritualität, Zivilreligion und Sinngebung aus den Daten (vgl. Bernts und Berghuijs 2016, S. 186).

Linda Woodhead (2017b), die in ihren Studien zu no religion zu ähnlichen Ergebnissen gelangt, zieht daraus Konsequenzen für die Forschung. Sie meint, dass die bisherigen in der Religionssoziologie entwickelten Forschungsinstrumente bei diesem Phänomen zu kurz greifen würden und empfiehlt eine Ausweitung der Forschung in den weiteren Bereich von Kultur und Werten. Eine ähnliche Empfehlung für die Religionspsychologie könnte lauten, sich nicht nur im Hinblick auf das Phänomen no religion noch stärker als bisher an einer (nicht kulturalistisch verkürzten) Handlungs- und Kulturpsychologie zu orientieren, wie sie u.a. von Hans Werbik und KollegInnen in Erlangen entwickelt und an verschiedenen Orten (weiter) ausgearbeitet wurde (vgl. z. B. Kölbl und Sieben 2018).

Funding Open access funding provided by University of Amsterdam.

Open Access Dieser Artikel wird unter der Creative Commons Namensnennung 4.0 International Lizenz veröffentlicht, welche die Nutzung, Vervielfältigung, Bearbeitung, Verbreitung und Wiedergabe in jeglichem Medium und Format erlaubt, sofern Sie den/die ursprünglichen Autor(en) und die Quelle ordnungsgemäß nennen, einen Link zur Creative Commons Lizenz beifügen und angeben, ob Änderungen vorgenommen wurden.

Die in diesem Artikel enthaltenen Bilder und sonstiges Drittmaterial unterliegen ebenfalls der genannten Creative Commons Lizenz, sofern sich aus der Abbildungslegende nichts anderes ergibt. Sofern das betreffende Material nicht unter der genannten Creative Commons Lizenz steht und die betreffende Handlung nicht nach gesetzlichen Vorschriften erlaubt ist, ist für die oben aufgeführten Weiterverwendungen des Materials die Einwilligung des jeweiligen Rechteinhabers einzuholen.

Weitere Details zur Lizenz entnehmen Sie bitte der Lizenzinformation auf http://creativecommons.org/ licenses/by/4.0/deed.de.

\section{Literatur}

Alter, Joseph S. 2006. Yoga at the Fin de Siècle: Muscular Christianity with a 'Hindu' Twist. The International Journal of the History of Sport 23(5):759-776.

Ammerman, Nancy T. 2014. Sacred Stories, Spiritual Tribes. Finding Religion in Everyday Life. Oxford: Oxford University Press.

Berger, Peter L. 2012. Further Thoughts on Religion and Modernity. Society 49(4):313-316.

Berger, Peter L. 2014. The Many Altars of Modernity. Toward a Paradigm for Religion in a Pluralist Age. Berlin: de Gruyter.

Berger, Peter L. 2017. Die zwei Pluralismen. In Zwei Pluralismen. Positionen aus Sozialwissenschaft und Theologie zu religiöser Vielfalt und Säkularität, Hrsg. Peter L. Berger, Silke Steets, und Wolfram Weiße, 17-27. Münster: Waxmann.

Berghuijs, Joantine. 2018. Meervoudig Religieus. Spirituele Openheid en Creativiteit onder Nederlanders. Amsterdam: Amsterdam University Press.

Bergunder, Michael. 2020. Umkämpfte Historisierung. In Wissen um Religion: Erkenntnis-Interesse. Epistemologie und Episteme in Religionswissenschaft und Interkultureller Theologie, Hrsg. Klaus Hock, 47-131. Leipzig: Evangelische Verlagsanstalt.

Bernts, Ton, und Joantine Berghuijs. 2016. God in Nederland 1966-2015. Utrecht: Ten Have.

15 Die deutsche Übersetzung dieses Zitats ist von mir. 
Bohnsack, Ralf. 2000. Rekonstruktive Sozialforschung. Eine Einführung in Methodologie und Praxis qualitativer Forschung, 4. Aufl., Opladen: Leske + Budrich.

Boyer, Pascal. 2001. Religion Explained: The Evolutionary Origins of Religious Thought. New York: Basic Books.

Bruce, Steve. 2017. Secular Beats Spiritual. The Westernization of the Easternization of the West. Oxford: Oxford University Press.

Casanova, José. 2018. The Karel Dobbelaere Lecture: Divergent Global Roads to Secularization and Religious Pluralism. Social Compass 65(2):187-198.

Centraal Bureau voor Statistiek. 2020. Statistische Trends. https://www.cbs.nl/nl-nl/longread/statistischetrends/2020/religie-in-nederland/2-ontwikkelingen-in-religieuze-betrokkenheid. Zugegrriffen: 20. September 2021.

Cipriani, Roberto. 2017. Diffused Religion: Beyond Secularization. London: Palgrave Macmillan.

Davie, Grace. 2007. Vicarious Religion: A Methodological Challenge. In Everyday Religion: Observing Modern Religious Lives, Hrsg. Nancy T. Ammerman, 21-35.

Evans-Pritchard, Edward E. 1937. Witchcraft, Magic, and Oracles among the Azande. Oxford: Clarendon Press.

Fedele, Anna, und Kim E. Knibbe. 2020. Secular Societies, Spiritual Selves? The Gendered Triangle of Religion, Secularity and Spirituality. London: Routledge.

Foe-Aman, Germaine. 2021. Religieuze Knutselaars en Spirituele Nomaden. Onderzoek naar Spirituele Motieven van Yoga Beoefenaars. Bachelorscriptie Religiewetenschappen. : Universiteit van Amsterdam.

Gendler, Tamar S. 2008. Alief and Belief. The Journal of Philosophy 105:634-663.

Habermas, Jürgen. 1985. Die neue Unübersichtlichkeit. Frankfurt am Main: Suhrkamp.

Heelas, Paul. 2014. On Transgressing the Secular: Spiritualities of Life, Idealism, Vitalism. In New Age Spirituality. Rethinking Religion, Hrsg. Steven J. Sutcliffe, Ingvild S. Gilhus, 66-83. London: Routledge.

Heelas, Paul, und Linda Woodhead. 2005. The Spiritual Revolution: Why Religion is Giving Way to Spirituality. Oxford: Blackwell.

Hense, Elisabeth, Frans Jespers, und Peter Nissen. 2014. Present-day Spiritualities: Contrasts and Overlaps. Leiden: Brill.

Kahnemann, Daniel. 2012. Thinking, Fast and Slow. London: Penguin Books.

Knoblauch, Hubert. 2009. Populäre Religion. Auf dem Weg in eine spirituelle Gesellschaft. Frankfurt am Main: Campus.

Kochinka, Alexander. 2008. Psychisches Geschehen im Tagebuch. Kulturpsychologische Fallstudien. Weilerswist: Velbrück.

Kölbl, Carlos, und Anna Sieben (Hrsg.). 2018. Stichwörter zur Kulturpsychologie. Gießen: PsychosozialVerlag.

Lanman, Jonathan. 2016. An Order of Mutual Benefit: A Secular Age and the Cognitive Science of Religion. In Working with A Secular Age: Interdisciplinary Reflections on Charles Taylor's Master Narrative, Hrsg. Florian Zemmin, Colin Jager, und Guido Vanheeswijk, 71-92. Berlin: de Gruyter.

Lee, Lois. 2015. Recognizing the Non-Religious. Reimagining the Secular. Oxford: University Press.

Legare, Christine H., und Susan A. Gelman. 2008. Bewitchment, Biology, or Both: The Co-existence of Natural and Supernatural Explanatory Frameworks Across Development. Cognitive Science 32(4):607-642.

Lerner, Melvin J. 1980. The Belief in a Just World. A Fundamental Delusion. New York: Plenum Press.

Norenzayan, Ara. 2016. Theodiversity. Annual Review of Psychology 67:465-488.

Pollack, Detlef, und Gergely Rosta. 2017. Religion and Modernity: An International Comparison. Oxford: Oxford University Press.

Popp-Baier, Ulrike. 2018. Religion. In Stichwörter zur Kulturpsychologie, Hrsg. Carlos Kölbl, Anna Sieben, 341-346. Gießen: Psychosozial-Verlag.

Stolz, Jörg. 2020. Secularization Theories in the Twenty-First Century: Ideas, Evidence, and Problems. Social Compass 67(2):282-308.

Straub, Jürgen, und Dilek A. Tepeli. 2021. Verletzungsverhältnisse, interreligiöse Konflikte und Abjektionen in multikulturellen Einwanderungsgesellschaften. Revisionen in der interdisziplinären Migrationsforschung. In Figurationen spätmoderner Lebensführung, Hrsg. Susanne Benzel, Katarina Busch, Benedikt Salfeld, und Julia Schreiber, 143-195. Wiesbaden: Springer VS.

Voas, David. 2009. The Rise and Fall of Fuzzy Fidelity in Europe. European Sociological Review 25(2):155-168. 
Wilkins-LaFlamme, Sarah. 2021. A Tale of Decline or Change? Working Toward a Complementary Understanding of Secular Transition and Individual Spiritualization Theories. Journal for the Scientific Study of Religion $0(0): 1-24$.

Woodhead, Linda. 2016. Intensified Religious Pluralism and De-differentiation: The British Example. Society 53(1):41-46.

Woodhead, Linda. 2017a. Pluralismus und der Aufstieg der „Nones“. In Zwei Pluralismen. Positionen aus Sozialwissenschaft und Theologie zu religiöser Vielfalt und Säkularität, Hrsg. Peter L. Berger, Silke Steets, und Wolfram Weiße, 127-145. Münster: Waxmann.

Woodhead, Linda. 2017b. 2016 Paul Hanly Furfey Lecture. The Rise of "No Religion": Towards an Explanation. Sociology of Religion 78(3):247-262.

Yang, Fenggang. 2012. Religion in China: Survival and Revival under Communist Rule. New York: Oxford University Press.

Yang, Fenggang. 2014. Agency-Driven Secularization and Chinese Experiments in Multiple Modernities. In The Many Altars of Modernity. Toward a Paradigm for Religion in a Pluralist Age, Hrsg. Peter L. Berger, 123-140. Berlin: de Gruyter.

Zulehner, Paul M. 2011. Verbuntung. Kirchen im weltanschaulichen Pluralismus, Religion im Leben der Menschen 1970-2010. Ostfildern: Schwabenverlag.

Hinweis des Verlags Der Verlag bleibt in Hinblick auf geografische Zuordnungen und Gebietsbezeichnungen in veröffentlichten Karten und Institutsadressen neutral. 